\title{
The Urge to Merge: When Cellular Service Providers Pool Capacity
}

\author{
Sha Hua, Pei Liu and Shivendra S. Panwar*
}

\begin{abstract}
As cellular networks are turning into a platform for ubiquitous data access, cellular operators are facing a severe data capacity crisis due to the exponential growth of traffic generated by mobile users. In this work, we investigate the benefits of sharing infrastructure and spectrum among two cellular operators. Specifically, we provide a multi-cell analytical model using stochastic geometry to identify the performance gain under different sharing strategies, which gives tractable and accurate results. To validate the performance using a realistic setting, we conduct extensive simulations for a multi-cell OFDMA system using real base station locations. Both analytical and simulation results show that even a simple cooperation strategy between two similar operators, where they share spectrum and base stations, roughly quadruples capacity as compared to the capacity of a single operator. This is equivalent to doubling the capacity per customer, providing a strong incentive for operators to cooperate, if not actually merge.
\end{abstract}

\section{INTRODUCTION}

The recent commercialization and popularization of $3 \mathrm{G}$ networks have significantly enhanced the mobile users' capability of making use of ubiquitous data access. However, as users "mobilize" all of their communication activities, from streaming video to cloud computing tasks, cellular operators are facing a severe data capacity crisis. A recent study of Cisco predicts that mobile data traffic will skyrocket by a factor between $25 \mathrm{X}$ to $50 \mathrm{X}$ by 2015 [1]. Such explosive data traffic growth will overload the cellular infrastructure and result in either poor or expensive service to the subscribers. To address this challenge, the most straightforward solutions, such as adding cells and increasing spectrum have become either expensive or inefficient options.

Cooperation among operators is yet another solution which has not drawn much attention from researchers. As cellular operators are devoting most of efforts in expanding their respective networks due to the urgency of the data capacity crisis and market competition, it has become apparent that there exist huge variations in spectrum usage, channel quality and coverage in different operators' networks [2]. Such diversity generates plenty of cooperation opportunities, which can be exploited to improve their network performance, spectrum efficiency and user experience.

One motivating example is shown in Figure 1 Two operators, Operator 1 and Operator $2\left(\mathrm{OP}_{1}\right.$ and $\mathrm{OP}_{2}$ for short), provide cellular service in one area. We assume their base stations (BSs) are deployed following a hexagonal layout (solid and dotted lines, respectively) and are interweaved. Without cooperation,

\footnotetext{
*The authors are with Polytechnic Institute of New York University, Brooklyn, NY, 11201, USA. Emails: shua01@students.poly.edu; pliu@poly.edu; panwar@catt.poly.edu
}

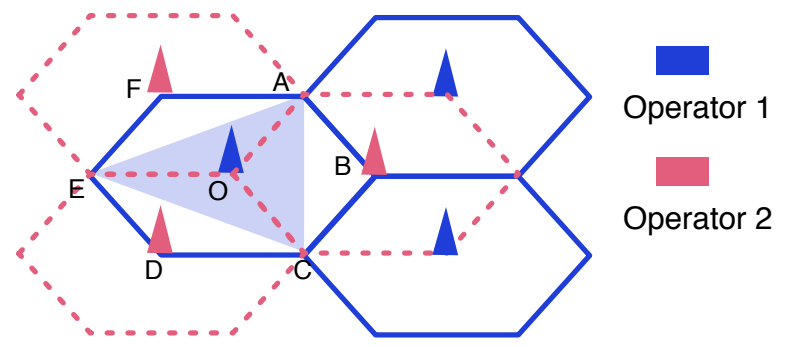

Fig. 1. Motivating scenario of cooperation between cellular operators.

the two operators will serve their own users solely using their own spectrum bands. The edge users, such as those at points $\mathrm{B}, \mathrm{D}$ and $\mathrm{F}$ in the left cell of $\mathrm{OP}_{1}$, will experience bad channel condition and strong inter-cell interference. However, if the two operators cooperate by allowing their users to flexibly connect to any $\mathrm{BS}$ that is closest to them, users of $\mathrm{OP}_{1}$ at $\mathrm{B}, \mathrm{D}$ and $\mathrm{F}$ will be served by the BSs of $\mathrm{OP}_{2}$ and have excellent channels to the BSs. Generally, the users of $\mathrm{OP}_{1}$ in the area $\triangle A B C$, $\triangle E C D$ and $\triangle E F A$ will enjoy performance gains; these are mostly edge users with low data rates. A similar effect happens to the users of $\mathrm{OP}_{2}$ as well, such as those in the area $\triangle A O C$. As we can see, simple cooperation improves the capacity of both operators and results in a win-win situation. Depending on the levels of synergy, the operators can share each other's infrastructure, spectrum, or even merge into a single operator. Interestingly, in AT\&T's proposed acquisition of T-Mobile, one of the reasons cited is the resulting increase in capacity [3].

In this paper, we focus on studying the benefits brought by different levels of cooperation among cellular operators. We are interested in answering the following questions: In what ways can the operators cooperate? How much performance improvement can their cooperation bring? Only a few recent papers address this issue [4][5][6]. In [4], Chandramani et al. optimize the operators' aggregate payoffs given BS locations and user rates, and use game theory to discuss how to share the profits. Supratim et al. in [5] develop a user choice algorithm with network information provided by the operators. Peng et al. in [6] focus on spectrum-based cooperation, and form a group bargaining model based on the demand on each BS.

However, all the above papers require cross-layer optimization based on the instantaneous network status. They do not characterize the long-term network capacity improvement for a large-scale multi-cell deployment. Our research fills this gap and provides a tractable and accurate model for quantifying the gains under different cooperation strategies. To validate our model, we have collected a set of real BS location data and provide performance results using real-world layouts under 
an Orthogonal Frequency Division Multiple Access (OFDMA) system. This aspect also has not yet been examined before. We expect our paper to give an insight into the benefit of cooperation and guide operators considering a range of cooperation options. Specifically the contribution of the paper is two-fold:

- We present an analytical model for identifying the performance gain from cooperation among cellular operators. It provides a tractable and reasonably accurate model for average user rate/throughput under a multi-cell environment.

- We perform extensive simulations using real BS locations and OFDMA resource allocation algorithms. It validates the advantage of operator cooperation in a realistic setting.

The remainder of the paper is organized as follows. Section II presents two modes of cooperation and the analytical model. In Section III, we provide an OFDMA resource allocation algorithm. The performance evaluation is presented in Section IV. Finally, the paper is concluded in Section V.

\section{Analytical Modeling}

In this section, we derive an analytical model to evaluate the network performance when the cellular operators cooperate. We consider two strategies, with different levels of cooperation, that we envisage the cellular operators may adopt:

1) FLEXROAM (short for "flexible roaming"): the cellular operators allow their users to freely connect to a BS of either operator that provides the best signal strength. It will require an update in the signaling protocols to facilitate this.

2) MERGER: in addition to FLEXROAM, all the BSs of an operator can reuse the spectrum of its cooperating operators. This may be the result of a full merger of the two operators, but does not preclude a mutual business agreement short of a full merger.

Note that FLEXROAM and MERGER can also be offered to users by a mobile virtual network operator, or MVNO, which can purchase wholesale use of the infrastructure from the two operators. We further use NOCOOP to refer to the scheme that the two operators do not cooperate.

Cellular networks are traditionally modeled by assuming the BSs are placed following a hexagonal layout [7]. However, these models have long suffered from being both intractable and highly idealized. Recently, a general model based on stochastic geometry was proposed in [8], which provides tractable ways to evaluate network performance considering inter-cell interference and fading. Our analytical modeling follows the methods in [8], and provides tractable results of the performance under the various modes of operators' cooperation.

We will mainly focus on the user's average ergodic rate and average throughput, which are important metrics for benchmarking the cellular system. We assume there are two operators, $\mathrm{OP}_{1}$ and $\mathrm{OP}_{2}$, coexisting in the network. Our models can be easily extended to include more than two operators, and to derive other performance metrics, such as the distribution of user's Signal-to-Interference-plus-Noise Ratio (SINR).

We assume the locations of BSs of $\mathrm{OP}_{1}$ and $\mathrm{OP}_{2}$ follow independent Poisson point processes $\Phi_{1}$ and $\Phi_{2}$ with densities $\lambda_{1}$ and $\lambda_{2}$, respectively. The model drawn from such a random deployment is shown to be about as accurate as the standard grid model, compared to an actual cellular network [8]. Further we use $W_{i}$ to denote channel bandwidths used by the BSs of $\mathrm{OP}_{i}, i \in\{1,2\}$. We consider the downlink performance of the system. Without losing generality, we assume a typical user is located at the origin. If it is connected to the $\mathrm{BS} b_{0}$ of $\mathrm{OP}_{i}$, its SINR can be expressed as:

$$
\operatorname{SINR}_{i}=\frac{P_{t} h_{b_{0}} r_{b_{0}}^{-\alpha}}{N_{0} W_{i}+P_{t} \sum_{b \in \Phi_{i} \backslash b_{0}} g_{b} r_{b}^{-\alpha}},
$$

where $P_{t}$ is the fixed transmission power of all the BSs, $N_{0}$ is the noise power density. The distance between the user and BS $b_{0}$ is $r_{b_{0}}$, and the channel fading is $h_{b_{0}}$. The user's distances to other interfering BSs $b$ in $\Phi_{i}$ are $r_{b}$ and the corresponding channel fadings are $g_{b} . \alpha$ is the path loss exponent. We assume all the fadings are Rayleigh fading with mean 1.

\section{A. Average User Ergodic Rate}

Following the proofs in [8], when the two operators do not cooperate and run their service as is, the average ergodic rate in the downlink for a typical user of $\mathrm{OP}_{i}$ 's is given by:

$$
\begin{aligned}
& R_{\text {NOCOOP }}\left(W_{i}, \lambda_{i}\right)=\mathbb{E}\left[\ln \left(1+\operatorname{SINR}_{i}\right)\right] \\
= & \int_{r>0} e^{-\pi \lambda_{i} r^{2}} \int_{t>0} e^{-\frac{N_{0} W_{i} r^{\alpha}\left(e^{t}-1\right)}{P_{t}}} F\left(\lambda_{i}\right) \mathrm{d} t 2 \pi \lambda_{i} r \mathrm{~d} r(2)
\end{aligned}
$$

where

$$
F\left(\lambda_{i}\right)=\exp \left(-\pi \lambda_{i} r^{2}\left(e^{t}-1\right)^{2 / \alpha} \int_{\left(e^{t}-1\right)^{-2 / \alpha}}^{\infty} \frac{1}{1+x^{\alpha / 2}} \mathrm{~d} x\right) .
$$

Eq. (2) and (3) can be computed numerically and are used for performance comparisons in this paper. Next we examine the performance under cooperation strategies. When $\mathrm{OP}_{1}$ and $\mathrm{OP}_{2}$ share infrastructure following the FLEXROAM strategy, we have the following theorem:

Theorem 1: Under FLEXROAM strategy, for a typical user, the average ergodic rate is given by:

$$
\begin{aligned}
& R_{F L E X R O A M}(\mathbf{W}, \boldsymbol{\lambda}) \\
= & \frac{\lambda_{1}}{\lambda_{1}+\lambda 2} R_{1}\left(W_{1}, \boldsymbol{\lambda}\right)+\frac{\lambda_{2}}{\lambda_{1}+\lambda 2} R_{2}\left(W_{2}, \boldsymbol{\lambda}\right),
\end{aligned}
$$

where

$$
\begin{aligned}
R_{i}\left(W_{i}, \boldsymbol{\lambda}\right)= & \int_{r>0} e^{-\pi\left(\lambda_{1}+\lambda_{2}\right) r^{2}} \int_{t>0} e^{-\frac{N_{0} W_{i} r^{\alpha}\left(e^{t}-1\right)}{P_{t}}} . \\
& F\left(\lambda_{i}\right) \mathrm{d} t 2 \pi\left(\lambda_{1}+\lambda_{2}\right) r \mathrm{~d} r .
\end{aligned}
$$

and $F\left(\lambda_{i}\right)$ is given by Eq. (3). $\mathbf{W}=\left\{W_{1}, W_{2}\right\}$ and $\boldsymbol{\lambda}=$ $\left\{\lambda_{1}, \lambda_{2}\right\}$ are the vectors for bandwidths and BS densities.

Proof: We use $r$ to denote the distance between the user and its associated BS. Under FLEXROAM, the user will connect to the closest BS from either of the operators that gives the best average signal strength. The union of all the BSs is still a Poisson point process $\Phi$ with density $\lambda_{1}+\lambda_{2}$. The cumulative density function (CDF) of the distance $r$ is then:

$$
\begin{aligned}
F_{r}(R) & =\operatorname{Pr}[r \leq R]=1-\operatorname{Pr}[r>R] \\
& =1-\operatorname{Pr}[\text { no BS from any operator closer than } R] \\
& =1-e^{-\left(\lambda_{1}+\lambda_{2}\right) \pi R^{2}} .
\end{aligned}
$$


Thus the probability density function (PDF) of $r$ is:

$$
f_{r}(r)=\frac{\mathrm{d} F_{r}(r)}{\mathrm{d} r}=e^{-\left(\lambda_{1}+\lambda_{2}\right) \pi r^{2}} 2 \pi\left(\lambda_{1}+\lambda_{2}\right) r .
$$

Moreover, we have

$$
R_{F L E X R O A M}(\mathbf{W}, \boldsymbol{\lambda})=\int_{r>0} f_{r}(r) \mathbb{E}_{h, g, \Phi}(\ln (1+\mathrm{SINR})) \mathrm{d} r .
$$

We further use $X_{i}=1$ to represent the event that the user is associated to the BS of $\mathrm{OP}_{i}$. Note $X_{i} \in\{0,1\}, \forall i=1,2$ and $X_{1}+X_{2}=1$. According to the rule of total probability,

$\mathbb{E}_{h, g, \Phi}(\ln (1+\mathrm{SINR}))=\sum_{i=1,2} \mathbb{E}_{h, g, \Phi_{i}}\left(\ln \left(1+\operatorname{SINR}_{i}\right)\right) \operatorname{Pr}\left[X_{i}=1\right]$,

wherein, $\operatorname{SINR}_{i}$ is given by Eq. (1). Using $D_{i}$ to represent the user's distance to the closest $\mathrm{BS}$ from $\mathrm{OP}_{i}$, in a manner similar to Eq. (7), the CDF of $D_{i}$ is $f_{D_{i}}(r)=e^{-\lambda_{i} \pi r^{2}} 2 \pi \lambda r$. We have:

$$
\begin{aligned}
& \operatorname{Pr}\left[X_{1}=1\right]=\operatorname{Pr}\left[D_{1}<D_{2}\right] \\
= & \int_{0}^{\infty} \int_{r_{1}}^{\infty} e^{-\lambda_{2} \pi r_{2}^{2}} 2 \pi \lambda_{2} r_{2} e^{-\lambda_{1} \pi r_{1}^{2}} 2 \pi \lambda_{1} r_{1} \mathrm{~d} r_{2} \mathrm{~d} r_{1} \\
= & \lambda_{1} /\left(\lambda_{1}+\lambda_{2}\right) .
\end{aligned}
$$

Similarly, we have $\operatorname{Pr}\left[X_{2}=1\right]=\lambda_{2} /\left(\lambda_{1}+\lambda_{2}\right)$. Plugging Eq. (9) and (10) into (8), and following the rest of the steps as in Appendix B of [8] completes the proof.

We next discuss the average ergodic rates when the MERGER strategy is employed. Under this scheme, each BS reuses the spectrum owned by the other operators, e.g., they can operate on the whole spectrum $W_{1}+W_{2}$. As a result, the BSs from both operators interfere with each other. We then have the following corollaries from Eq. (2) and (3):

Corollary 1: Under the MERGER strategy, for a typical user, the average ergodic rate is:

$$
R_{M E R G E R}(\mathbf{W}, \boldsymbol{\lambda})=R_{\text {NOCOOP }}\left(W_{1}+W_{2}, \lambda_{1}+\lambda_{2}\right) .
$$

Proof: This is same as the single operator case with BSs of density $\lambda_{1}+\lambda_{2}$ operate on bandwidth $W_{1}+W_{2}$. We then have the corollary following Eq. (2) and (3).

\section{B. Average User Throughput}

We will now determine the average throughput for a typical user under FLEXROAM and MERGER. We use $\eta_{i}$ to denote the subscriber density of $\mathrm{OP}_{i}, i \in\{1,2\}$. Generally, subscriber density is far larger than the macrocell BS density, thus we assume $\eta_{i} \geq \lambda_{j}, \forall i, j \in\{1,2\}$.

According to the Law of Large Numbers, in NOCOOP, if the two operators serve their users independently, the number of users in each cell of $\mathrm{OP}_{i}$ will be approximately $\frac{\eta_{i} S}{\lambda_{i} S}=\frac{\eta_{i}}{\lambda_{i}}$, where $S$ is the total area under consideration. We further assume that the scheduling decisions on the BSs ensure proportional fairness, which is a common design objective of all current and next generation cellular systems. With proportional fairness, the average throughput of a user from $\mathrm{OP}_{i}$ is its rate divided by the number of users in the cell. Under NOCOOP scheme, it is:

$$
\operatorname{Th}_{\text {NOCOOP }}\left(W_{i}, \lambda_{i}, \eta_{i}\right)=R_{\text {NOCOOP }}\left(W_{i}, \lambda_{i}\right) \cdot \frac{\lambda_{i}}{\eta_{i}},
$$

Moreover, under FLEXROAM and MERGER strategy, the number of users in each cell converges to $\frac{\eta_{1}+\eta_{2}}{\lambda_{1}+\lambda_{2}}$. Therefore, the average throughput for a typical user is:

$$
\begin{gathered}
T h_{F L E X R O A M}(\mathbf{W}, \boldsymbol{\lambda}, \boldsymbol{\eta})=R_{F L E X R O A M}(\mathbf{W}, \boldsymbol{\lambda}) \frac{\lambda_{1}+\lambda_{2}}{\eta_{1}+\eta_{2}} \\
=\frac{1}{\eta_{1}+\eta_{2}}\left(\lambda_{1} R_{1}\left(\lambda_{1}, \lambda_{2}\right)+\lambda_{2} R_{2}\left(\lambda_{1}, \lambda_{2}\right)\right) .
\end{gathered}
$$

where $\boldsymbol{\eta}=\left\{\eta_{1}, \eta_{2}\right\}$. Finally, under the MERGER strategy, the average throughput for a user is:

$$
T h_{M E R G E R}(\mathbf{W}, \boldsymbol{\lambda}, \boldsymbol{\eta})=R_{M E R G E R}(\mathbf{W}, \boldsymbol{\lambda}) \frac{\lambda_{1}+\lambda_{2}}{\eta_{1}+\eta_{2}} .
$$

Compared to the traditional hexagonal model that requires complex system-level Monte-Carlo simulations, our analytical model can be computed using a numerical tool in a short time.

\section{An OFDMA-BASEd Multi-Cell System}

In the previous section, we described an analytical model for evaluating the network performance under different cooperation strategies of the operators. However, the model simplifies the real system in three aspects: 1) it assumes Poisson random BS deployment; 2) it uses the entire spectrum without considering subchannelization; 3) it assumes perfect resource allocation following the proportional fairness criterion.

Due to the above constraints, it is still good to validate what the network performance will be in a practical multi-cell system under the various cooperation strategies. To evaluate the benefits of operator cooperation in realistic system, we plan to combine real BS location data and practical user-subchannel scheduling algorithms. As OFDMA is widely adopted by all the next-generation cellular standards (WiMAX, LTE) to divide the spectrum into subchannels, in this section, we present a low complexity OFDMA multi-cell resource allocation algorithm. Results with real BS locations are given in the next section.

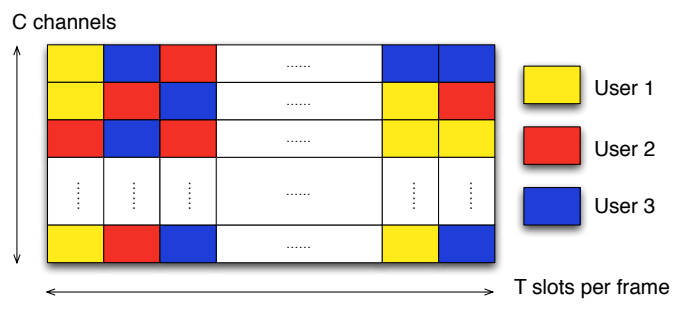

Fig. 2. A typical OFDMA frame in a temporal-frequency domain.

A typical OFDMA system partitions the radio resource in both frequency and time domains as shown in Figure 2. The total bandwidth is divided into $C$ orthogonal subchannels, and one frame is divided into $T$ time slots. A subchannel and time slot combination, termed a tile, is the minimum unit for resource allocation. In one BS, a tile can only be assigned to one user to avoid intra-cell interference. For each BS $b$, we use $\mathcal{M}_{b}$ to denote the set of users associated with it. Thus there are totally $\left|\mathcal{M}_{b}\right|$ users in this cell. For a specific BS, 
$\mathcal{M}_{b}$ may vary depending on the different cooperation strategies the operators use. Specifically, in NOCOOP, $\mathcal{M}_{b}$ are the users from the same operator of $b$ that are connected to $b$, whereas in FLEXROAM and MERGER, $\mathcal{M}_{b}$ include the users from the other operators that treat $b$ as the best BS. In addition, the number of subchannels $C$ may also vary according to the cooperation strategy. It will increase in MERGER as each BS reuses the spectrum owned by the other operators.

In the multi-cell scenario, neighboring cells may reuse the same tile depending on the inter-cell interference. As a result, the optimal multi-cell resource allocation problem is combinatoric and generally regarded as an NP-hard problem. Only heuristic methods exist so far [9][10][11]. Next, we present a centralized greedy algorithm to achieve a sub-optimal solution with low computational complexity. It assumes the users' channel state information is available at a Radio Network Controller (RNC), which coordinates all the BSs. Note that since our purpose is to quantify the gain brought by the operator cooperation, designing more practical algorithms is beyond our scope. Our algorithm is generalized from the ones in [9][10]. It runs for each frame and aims to maximize the total utilities of all the users throughout all the cells in a frame. The utility of user $i$ is defined as $U\left(R_{i}\right)=\log \left(R_{i}\right)$, with $R_{i}$ being its data rate in the current frame. Using log function as the utility has been proved to be equivalent to finding a proportional fair solution [12]. Assuming there are $B$ BSs and $N$ users, the complexity of the algorithm is $O(T C B N)$.

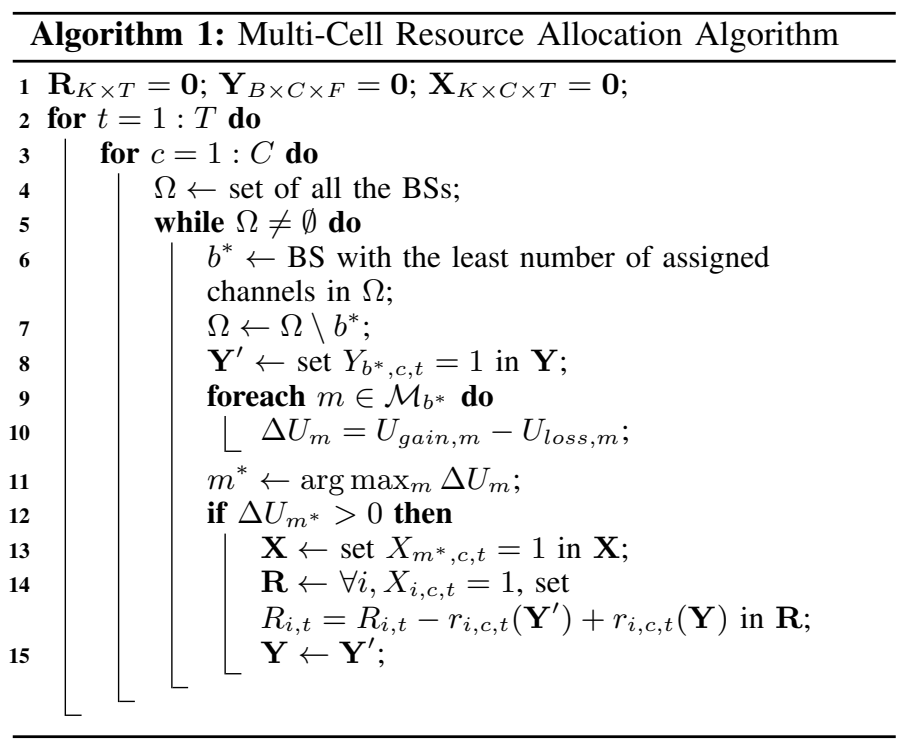

As shown in Algorithm 1, Line 1 initializes the matrices that contain the allocation results. Each entry $R_{i, t}$ in $\mathbf{R}$ records the aggregate data rate of user $i$ up to the slot $t . \mathbf{X}$ and $\mathbf{Y}$ are boolean matrices. The entry $Y_{b, c, t}=1$ in $\mathbf{Y}$ if BS $b$ is transmitting on subchannel $c$ in slot $t$. The entry $X_{i, c, t}=1$ in $\mathbf{X}$ indicates that the user $i$ is receiving on subchannel $c$ in slot $t$. The subchannels are allocated sequentially (Line 3 ), and for each one, we check each BS for whether it should transmit on this subchannel in the current slot. To maintain fairness, the BSs with fewer number of assigned subchannels are given higher priority (Line 6-7). Our algorithm is marginal utility driven. For the current BS $b^{*}$, Line 9-13 picks the user with the maximal marginal utility $\Delta U$. For each user $m$ associated with $b^{*}, \Delta U_{m}$ is defined as the difference between the utility increase $U_{\text {gain, } m}$ by scheduling user $m$ and the total utility loss of other users on the same subchannel $U_{\text {loss }, m}$ due to the increased interference (Line 10). $U_{\text {gain }, m}$ and $U_{\text {loss }, m}$ can be evaluated by:

$$
\begin{array}{r}
U_{\text {gain }, m}=U\left[R_{m, t}+r_{m, c, t}\left(\mathbf{Y}^{\prime}\right)\right]-U\left(R_{m, t}\right) \\
U_{\text {loss }, m}=\sum_{i, X_{i, c, t}=1}\left\{U\left(R_{i, t}\right)-U\left[R_{i, t}-r_{m, c, t}(\mathbf{Y})+r_{m, c, t}\left(\mathbf{Y}^{\prime}\right)\right]\right\}
\end{array}
$$

$\mathbf{Y}^{\prime}$ is the matrix assuming BS $b^{*}$ is transmitting on subchannel $c$ in slot $t$. The function $r_{m, c, t}(\mathbf{Y})$ is the rate of user $m$ on subchannel $c$ in slot $t$ based on the allocation matrix $\mathbf{Y}$. We assume the transmit power is equally split across the subchannels on each BS. Considering the inter-cell interference, $r_{m, c, t}(\mathbf{Y})$ is defined as:

$r_{m, c, t}(\mathbf{Y})=W_{C} \log _{2}\left(1+\frac{P_{t} / C \cdot h_{b_{0}, m}^{c} d_{b_{0}, m}^{-\alpha}}{N_{0} W_{C}+\sum_{b \neq b_{0}, Y_{b, c, t}=1} h_{b, m}^{c} d_{b, m}^{-\alpha}}\right)$,

where $W_{C}=W / C$ is the bandwidth of a subchannel, $b_{0}$ is the BS user $m$ associates to. $h_{b, m}^{c}$ is the subchannel fading between BS $b$ and user $m$ on subchannel $c$, and $d_{b, m}$ is the distance between BS $b$ and user $m$. Finally, Line 12-15 update the matrices if the maximal marginal utility is positive.

\section{Performance Evaluation}

In this section, we assess the performance of our proposed cooperation strategies through two sets of simulations. In the first set, we numerically compute the expressions given by the analytical model described in Sec. II. To further show the results under practical settings, in the second set, we conduct simulations for the OFDMA system with the multi-cell resource allocation algorithm shown in Sec. III.

We have obtained precise coordinates for BSs from two major operators over a large suburban area near Washington D.C.. The $20 \times 20 \mathrm{~km}$ area we chose is shown in Figure 3 There are $16 \mathrm{BSs}$ from one operator and $13 \mathrm{BSs}$ from the other. We mark the BSs using red and blue to distinguish them. Their location information will be used for our simulation.

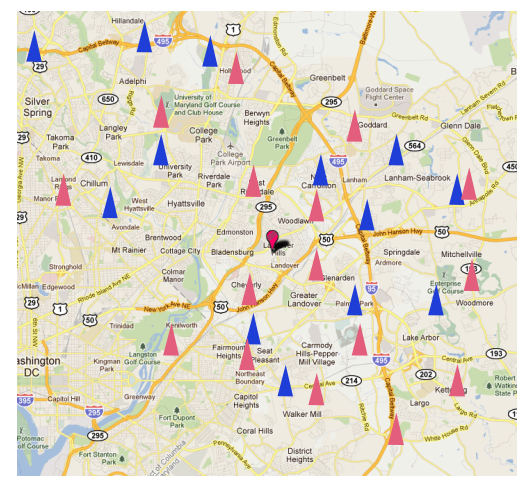

Fig. 3. A $20 \times 20 \mathrm{~km}$ view of the current BS deployment by two major cellular operators in the Washington D.C. area. 


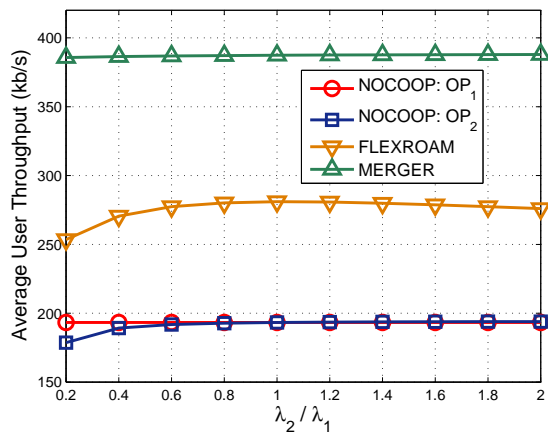

Fig. 4. The impact of BS density.

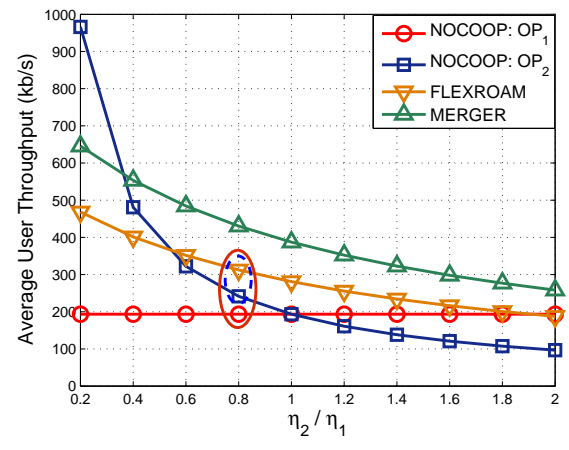

Fig. 5. The impact of user density.

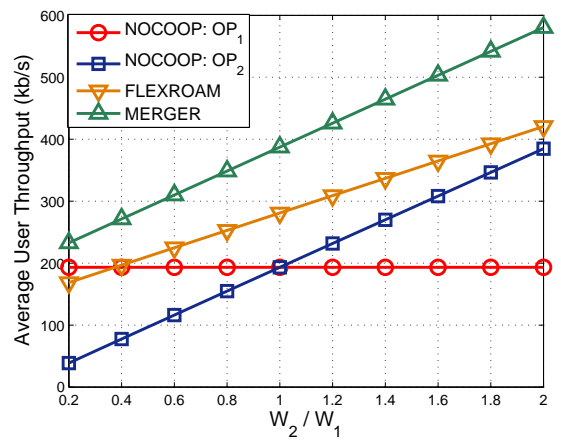

Fig. 6. The impact of bandwidth.

\section{A. Numerical Evaluations}

Here we numerically compute the results of our analytical model when different cooperation strategies are employed by the operators. We will focus on user's average throughput as it is a critical metric for evaluating cellular service. It is important to show how much improvement there is through operator cooperation, and how such an improvement varies when the two operators have different BS densities, user densities and bandwidths. We set the value of $\lambda_{1}$ in Eq. (12)-(14) as the red operator's BS density, e.g., $\lambda_{1}=16 / 400000000=4 * 10^{-8}$. $\lambda_{1}$ will be fixed in all the scenarios while $\lambda_{2}$ is left as an adjustable parameter. According to IEEE $802.16 \mathrm{~m}$ evaluation methodology document [13], we set the transmission power of the BSs as $P t=46 \mathrm{dBm}$, noise power density as $N_{0}=-174$ $\mathrm{dBm} / \mathrm{Hz}$ and the path loss exponent $\alpha=3.76$.

1) The Impact of BS Density: In this scenario, we set both $\mathrm{OP}_{1}$ and $\mathrm{OP}_{2}$ 's bandwidths to $W_{1}=W_{2}=10 \mathrm{MHz}$ according to [13]. And we let their respective user densities be $\frac{\eta_{1}}{\lambda_{1}}=$ $\frac{\eta_{2}}{\lambda_{2}}=100$. As a result, their spectrum resource and average number of users per cell are identical. Figure 4 shows the results by changing the BS density ratio $\lambda_{2} / \lambda_{1}$. As $\lambda_{2}$ changes from $0.2 \lambda_{1}$ to $2 \lambda_{1}$, the performance of $\mathrm{OP}_{2}$ under NOCOOP only slightly increases, since $\lambda$ does not influence the signalto-interference ratio and the system is interference limited. We can easily see cooperation brings significant benefits to both operators. When $\frac{\lambda_{2}}{\lambda_{1}}=1$, the two operators have 193.3 $\mathrm{kb} / \mathrm{s}$ average user throughput without cooperation. However, if they cooperate, FLEXROAM and MERGER strategies improve the average user throughput to $281.0 \mathrm{~kb} / \mathrm{s}$ and $387.4 \mathrm{~kb} / \mathrm{s}$ respectively, which is equivalent to a $45.4 \%$ and $100.4 \%$ increase, respectively. As $\lambda_{2}$ keeps increasing, the performance of FLEXROAM will slightly drop since the opportunistic diversity is reduced. However, FLEXROAM and MERGER still achieve a large performance gain for both operators within a wide range of BS density ratios.

2) The Impact of User Density: To explore the impact of user densities on the network performance of cooperation, we fix $\lambda_{2}=\lambda_{1}=4 * 10^{-8}$ and $W_{2}=W_{1}=10 \mathrm{MHz}$. Then we let $\eta_{1}=100 \lambda_{1}$ and vary $\eta_{2}$ from $0.2 \eta_{1}$ to $2 \eta_{1}$. This represents different user/BS ratio for $\mathrm{OP}_{2}$, e.g., when $\eta_{2}=0.2 \eta_{1}=20 \lambda_{2}$, on average there are only 20 users per cell. Figure 5 shows the results. As we can see, as $\eta_{2}$ increases, under NOCOOP, the average user throughput of $\mathrm{OP}_{2}$ drops drastically when more users are sharing the radio resource. In contrast, under FLEXROAM and MERGER the performance degrades gradually since users of $\mathrm{OP}_{2}$ can still opportunistically connect to the $\mathrm{BSs}$ of $\mathrm{OP}_{1}$. Moreover, when $\mathrm{OP}_{1}$ and $\mathrm{OP}_{2}$ 's user densities are different, they achieve different performance gains. For instance when $\frac{\eta_{2}}{\eta_{1}}=0.8$, under NOCOOP, $\mathrm{OP}_{1}$ and $\mathrm{OP}_{2}$ have $193.26 \mathrm{~kb} / \mathrm{s}$ and $241.58 \mathrm{~kb} / \mathrm{s}$ average user throughput. When FLEXROAM is adopted, the performance jumps to $312.21 \mathrm{~kb} / \mathrm{s}$, which gives $61.5 \%$ and $29.2 \%$ gains to $\mathrm{OP}_{1}$ and $\mathrm{OP}_{2}$, respectively (marked in the circles in the figure). Generally, FLEXROAM results in a win-win situation when $\frac{\eta_{2}}{\eta_{1}}$ ranges from around 0.5 to 2.0. MERGER has a larger win-win range, which is from 0.37 to beyond 2.0. When the difference between $\eta_{1}$ and $\eta_{2}$ is too large, cooperation may generate negative effects to one operator. For example, when $\frac{\eta_{2}}{\eta_{1}}=0.4$, $\mathrm{OP}_{2}$ loses performance under FLEXROAM. However, since we just are studying a simple roaming policy, such negative effects are expected to be avoided by adopting more advanced policies and incentive mechanisms.

3) The Impact of BS Bandwidth: In this scenario, we fix $\eta_{1}=\eta_{2}=100 \lambda_{1}=100 \lambda_{2}$. We fix $W_{1}=10 \mathrm{MHz}$, and change the ratio $W_{2} / W_{1}$. As we can see from Figure 6 , the performance of FLEXROAM, MERGER and $\mathrm{OP}_{2}$ under NOCOOP increases almost linearly as the total bandwidth increases. When $\frac{W_{2}}{W_{1}}>0.4$, FLEXROAM provides better performance for both $\mathrm{OP}_{1}$ and $\mathrm{OP}_{2}$. It enables more gain for the operator with less spectrum. MERGER achieves a larger gain for all the points in the figure.

\section{B. Performance with Real BS Locations}

To validate the numerical results in practice, we conducted the OFDMA-based simulations with real BS locations. We consider two cellular networks with BSs placed as in Figure 3 . Mobile devices are deployed uniformly in the $20 \mathrm{~km} \times 20 \mathrm{~km}$ area with a density of 100 users per cell for each operator. The system is assumed to be coordinated by the OFDMA resource allocation algorithm we proposed in Sec. III. Based on the IEEE $802.16 \mathrm{~m}$ evaluation methodology document [13], the main system parameters are summarized in Table If For the channel gain, we model the fast fading component as Rayleigh fading 
TABLE I

OFDMA SYSTEM PARAMETERS

\begin{tabular}{l|l}
\hline Number of subchannels & 32 \\
\hline Number of slots per frame & 60 \\
\hline BS Transmit Power & $46 \mathrm{dBm}$ \\
\hline Noise power spectrum density $N_{0}$ & $-174 \mathrm{dBm}$ \\
\hline Channel bandwidth & $10 \mathrm{MHz}$
\end{tabular}

with mean 1 . We also considered shadow fading and model it as a log-normal random variable with a standard deviation of $8 \mathrm{~dB}$. The overall effect of antenna gain, cable and penetration loss and noise figure is set to $0 \mathrm{~dB}$. Finally, the path loss at the distance $d$ is modeled as $L(d)_{d B}=17.39+3.76 \log _{10} d$.

Depending on the different cooperation strategies, the user set of each BS may be different. Under NOCOOP and FLEXROAM, each operator still aims to optimize the total utility of all the users connected to its own BSs, while under MERGER, we optimize the global utility of the two operators. We ran each simulation for 30 consecutive frames and we averaged the results of 5 simulation runs. Further, we plug the density values $\lambda_{1}=16 / 400000000=4 * 10^{-8}$, $\lambda_{2}=13 / 400000000=3.25 * 10^{-8}, \eta_{1}=100 \lambda_{1}, \eta_{2}=100 \lambda_{2}$ into our previous analytical model to resolve the numerical results for comparison purpose.

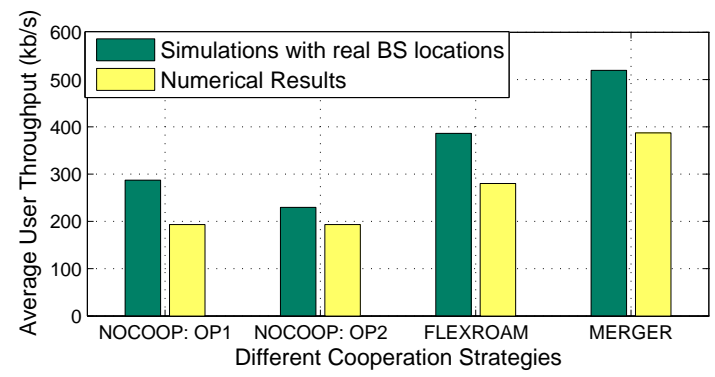

Fig. 7. network performance under OFDMA-based system.

Figure 7 shows the results. Under NOCOOP, the performance of $\mathrm{OP}_{2}$ is worse than $\mathrm{OP}_{1}$ since it has fewer $\mathrm{BSs}$ and the resulting average signal is weaker. Overall, the average throughput of all the users in the system under NOCOOP is around $263.8 \mathrm{~kb} / \mathrm{s}$. Cooperation substantially improves the performance of both the operators the real setting. Specifically, the user's average throughput increases to $386.24 \mathrm{~kb} / \mathrm{s}$ in FLEXROAM and $519.31 \mathrm{~kb} / \mathrm{s}$ in MERGER. These give $34.4 \%$ and $80.75 \%$ improvement to $\mathrm{OP}_{1}$ and $68.21 \%$ and $126.16 \%$ improvement to $\mathrm{OP}_{2}$ respectively. Moreover, our analytical model accurately captures this trend and provides very close improvement predictions in terms of percentage. OFDMA system generally offers better performance than the numerical results since it better exploits the user/frequency selectivity. Also, the analytical model is pessimistic since it assumes the random deployment of BSs, which is discussed in [8].

To further scrutinize the gain for the users, we draw the CDF of the average throughput of the users in one simulation run. Figure 8 shows the results in the domain of $0-200 \mathrm{~kb} / \mathrm{s}$. For NOCOOP, we use the statistics from all the users in the system. The curves validates the improvement result from cooperation.

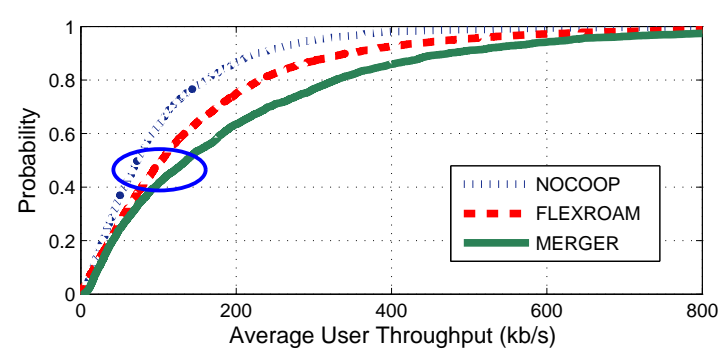

Fig. 8. user's average throughput CDF.

For example, the median value of user's average throughput increases by around $40 \%$ and 100\% under FLEXROAM and MERGER compared to NOCOOP.

\section{CONCLUSiOn}

This work investigates the potential benefits of cooperation among cellular operators. Using stochastic geometry, we provide a tractable analytical model to derive the average user rate and throughput under two cooperation strategies. With real base station locations, extensive simulations over multi-cell OFDMA system further validate the performance improvement. We show even a simple cooperation policy with modest changes to existing networks can achieve around 30\%-120\% capacity gains per customer under typical conditions.

\section{REFERENCES}

[1] "Global Mobile Data Traffic Forecast by Cisco." [Online]. Available: http://www.cisco.com/en/US/solutions/collateral/ns341/ns525/ ns537/ns705/ns827/white_paper_c11-520862.htmlf

[2] "Verizon iPhone Gets Better Coverage, Slower Data." [Online]. Available: http://www.wired.com/gadgetlab/2011/02/verizon-att-iphone-tests/

[3] "Acquisition of T-Mobile USA, Inc. by AT\&T Inc. - Description of Transaction, Public Interest Showing and Related Demonstrations," Filed with the Federal Communications Commission, April 2011.

[4] C. Singh, S. Sarkar, A. Aram, and A. Kumar, "Cooperative profit sharing in coalition-based resource allocation in wireless networks," Networking, IEEE/ACM Transactions on, to appear, 2011.

[5] S. Deb, K. Nagaraj, and V. Srinivasan, "MOTA: engineering an operator agnostic mobile service," in Proc. ACM MOBICOM, to appear, 2011.

[6] P. Lin, J. Jia, Q. Zhang, and M. Hamdi, "Cooperation among wireless service providers: opportunity, challenge, and solution," Wireless Communications, IEEE, vol. 17, no. 4, pp. 55-61, August 2010.

[7] T. S. Rappaport, Wireless Communications: Principles and Practice. Prentice Hall, 2002.

[8] J. G. Andrews, F. Baccelli, and R. K. Ganti, "A tractable approach to coverage and rate in cellular networks," Communications, IEEE Transactions on, to appear.

[9] I. Koutsopoulos and L. Tassiulas, "Cross-layer adaptive techniques for throughput enhancement in wireless OFDM-based networks," Networking, IEEE/ACM Transactions on, vol. 14, no. 5, pp. 1056-1066, October 2006.

[10] G. Li and H. Liu, "Downlink radio resource allocation for multi-cell OFDMA system," Wireless Communications, IEEE Transactions on, vol. 5, no. 12, pp. 3451-3459, December 2006.

[11] H. Zhang, L. Venturino, N. Prasad, P. Li, S. Rangarajan, and X. Wang, "Weighted sum-rate maximization in multi-cell networks via coordinated scheduling and discrete power control," Selected Areas in Communications, IEEE Journal on, vol. 29, no. 6, pp. 1214-1224, June 2011.

[12] F. P. Kelly, A. Maulloo, and D. Tan, "Rate control for communication networks: shadow prices, proportional fairness and stability," Journal of the Operational Research Society, vol. 49, pp. 237-252, 1998.

[13] I. 802.16m 08/004r1, "802.16m evaluation methodology document," March 2008. [Online]. Available: http://wirelessman.org/tgm/docs/ 80216m-08_004r1.pdf 